\title{
3-D MEASUREMENTS IN CONSTRUCTION ROBOTIZATION
}

\author{
Mikko Lindholm, Sakari Pieskä \\ Electronics Laboratory, VTT \\ Box 200, 90571 Oulu, Finland \\ Lauri Koskela \\ Laboratory of Urban Planning and Building Design, VTT \\ Itätuulenkuja 11, 02100 Espoo, Finland
}

VTT is an abbreviation for the Technical Research Centre of Finland.

\begin{abstract}
After an analysis of the needs and problems of measurements in construction, a general concept of using 3-D measurements is outlined. To study the suitability of advanced measurement techniques in construction, the flow of measurement data during automatic construction is examined. The general concept is adapted repair construction and and verified in a series of experiments.

The equipment in the first experiments has been a commercial measurement device. In a later state of the research, a 3-D vision system prototype (laser scanner) will be used in a repair construction task.

\section{INTRODUCTION}

The measurements of a construction project can be examined as a process, which starts from the measurements planning in the preliminary planning phase of a construction project and continues all the way to the as-built measurements of the completed building. The development towards computer integrated construction (CIC) makes measurement planning obligatory not only in the early phases of building but also in every phase of the CAD/CAM-process of prefabrication. The area of 3-D measurements is an interesting case because of the needs of automatic machines and the limitations of present measurements to 2-D and 1-D. It also involves complicated measurement methods and complex devices.

Advanced sensors and measurement devices will play an essential role in construction robotization. In the future, measurement planning could be supported with a knowledge-based system including sufficient a priori data for the use of both construction robots and advanced measurement devices. In addition, the utilization of advanced measurement devices and construction robots presupposes the existence of an integrated measurement system frame with a unified coordinate system and data base structure. The systematic nature of future measurements doesn't necessarily mean rigid rules for all 3-D measurements in construction. However, it means requirements to employ advanced $\mathrm{CIC}$ and $\mathrm{CAD}$ tools and to organize measurements.

The first goal of this paper is to describe the significance and needs for the automation of measurements in construction. In this connection a general concept for using 3-D measurements in construction is presented. The second aim is to analyse the suitability of advanced measurement techniques for construction applications. Also the most essential measurement requirements for construction robotics are presented. Thirdly, the paper reports and evaluates the results of preliminary experiments with 3-D
\end{abstract}


measurements. For this purpose repair construction has been chosen for a demonstration case and the general concept has been adapted to it. Finally targets for further research and development will be discussed.

\section{NEEDS AND SIGNIFICANCE OF MEASUREMENTS IN CONSTRUCTION}

The basis for the development of construction measurements must be a thorough knowledge of needs and current problems in the construction phases. To achieve that knowledge the evaluation of measurement requirements of four different construction areas was carried out by using a frame, which included evaluations of measurement needs, current measurement methods and problems, new solutions and the suitability of an integrated measuring system. The analyses used the results of a Finnish construction robotics feasibility study $/ 5 /$ in consultation with experts from the different laboratories of VTT and the results were reported in $/ 9 \%$

After the analysis was made, the idea of general measurements concept was examined. Because of the needs of automatic machines and the 2-D or 1-D nature of present measurement devices, 3-D measurements was chosen as a case study. The resulting concept is presented graphically in Fig. 1. The idea of computer integrated construction (CIC) is a starting point for this concept. The measurement process has been divided into planning and production phases. In some tasks in the different branches of construction these phases can intermix or some parts of the concept may be left out.

3D MEASUREMENTS IN COMPUTER INTEGRATED CONSTRUCTION

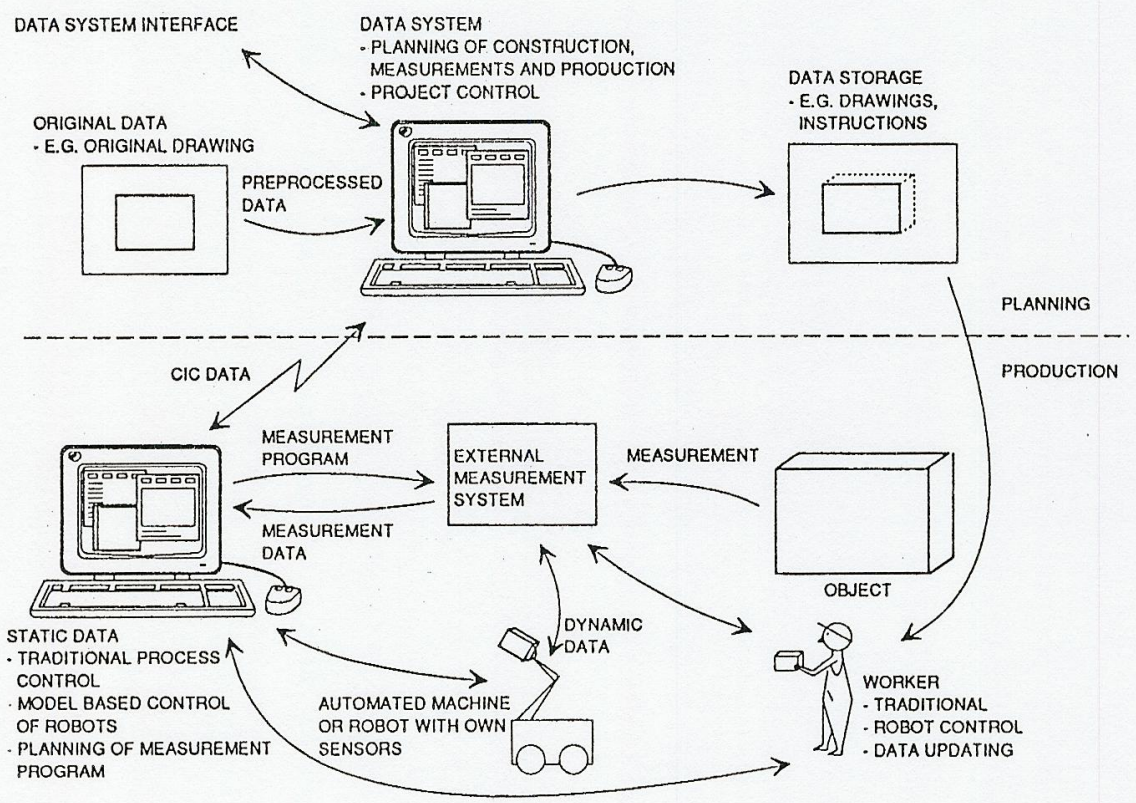

Figure 1. A concept for using 3-D measurements in construction.

In the centre of the concept is the data system. It involves the construction project control and it may have an interface to other systems. For example, a computerised repair construction project should have data on materials and prefabricated units needed. The data system takes original data, e.g. drawings of the framework, in order to plan measurements and to store data and print it in some other form. For example, paper 
drawings are a vital detail of man-machine interface. Production process is managed by a computer which controls the external measurement system and automated machines and robots. In order to apply this concept to reality, the 3-D measurement system has to be flexible and it must have an interface with the data system. The construction worker communicates, one way or another, with the whole data system.

The proposed concept for using 3-D measurements in construction must not be understood as a strict rule for standardisation, but more as a frame which can be adapted to many fields of construction. For example, often the external measurement system and the data system is one. In some cases a separate planning phase is not needed. This might be true in those repair construction tasks where original data simply doesn't exist. The main advantage of the concept is that it contains all the basic activities of 3-D measurements in construction arranged in a logical order. The suitability of the proposed concept has been verified by adapting this concept in repair construction and creating a working demonstration system of 3-D measurements.

\section{SUITABILITY OF ADVANCED MEASUREMENT TECHNIQUES FOR CONSTRUCTION APPLICATIONS}

Before the suitability of present measurement technology on construction sites is analysed, sources of data and the way of using the data in the process of construction must be known. When automatic construction machinery or construction robots are used, measurement data flow can be presented as in Fig. 2. Measurement data is obtained from materials, objects, tools and from the machines themself in three phases (preparation, working and inspection). This study gives the basis for further analysis.
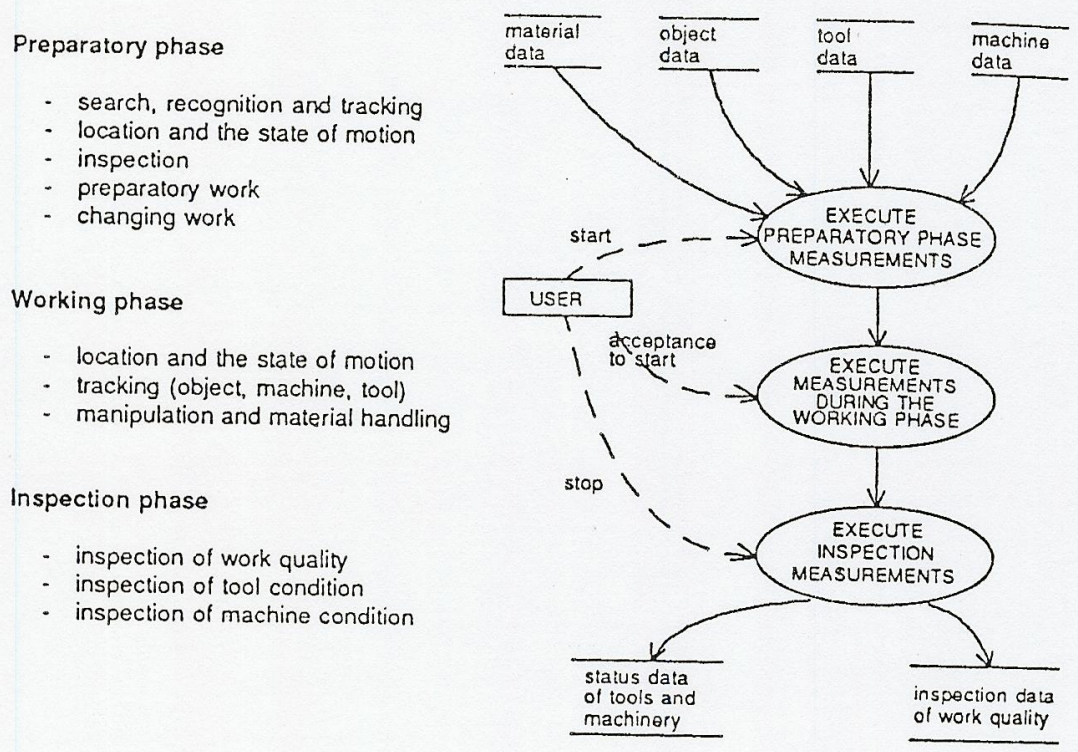

Figure 2. The flow of measurement data when using automatic consuuction machinery.

The required accuracies and resolutions of on-site measurements fall into several categories depending on the task, measurement range, environmental conditions, etc. For example, the position measurement accuracy required for a tower crane can be tens of centimeters while it is only a few millimeters for a masonry robot and even less for some tasks of an interior finishing robot. Resolutions of high-quality theodolites, tachymeters and lasers are approximately $1 \mathrm{~mm}$ when measurement ranges are under hundreds of meters. Therefore, it can be stated that with these devices preparatory phase 
measurements for construction robots can be carried out accurately enough in most construction applications. However, the measurement results are not usually available immediately and not in xyz-coordinates.

The real-time measurements needed during the working phase are a problem which cannot be solved without developing new methods for using the conventional construction measurement devices or applying and further developing the sensor technology used in other applications, e.g. in the manufacturing industry (see e.g. $/ 8,11$, 12/). Coordinate measurements will become increasingly important as future construction concentrates on prefabrication. One of the main prerequisites for the effective use of measuring instruments is to design and accomplish a unified coordinate system and data base structure. The most essential measuring requirements and potential solutions for construction robotics are collected in Table 1.

Table 1. The most essential measurement requirements for construction robotics.

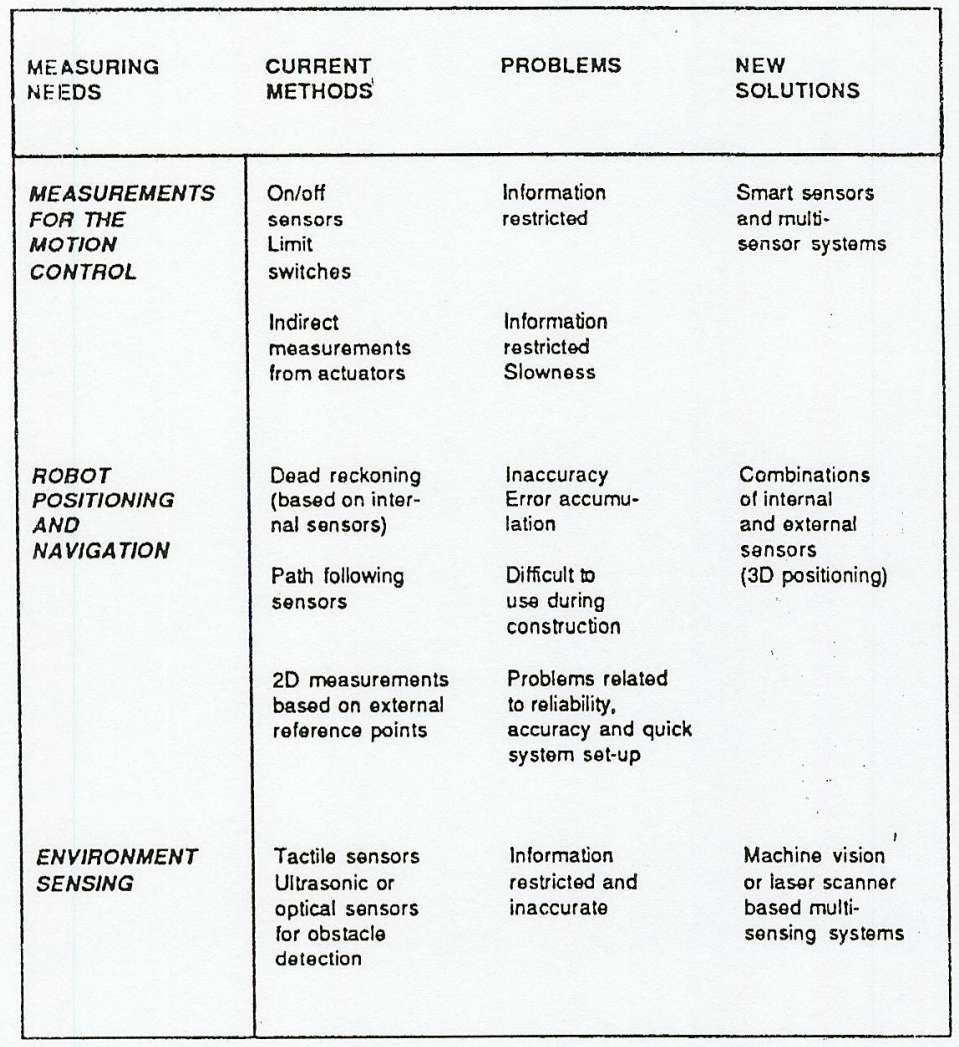

When the flow of measurement data and the requirements for certain construction tasks have been analysed the performance of present day technology can be examined. Level lasers, optical (electronic) distance meters, digital theodolites, tachymeters and socalled total stations (combined electronic theodolite and distance meter with a data collector) are examples of advanced, on-site measurement devices, which are substituting traditional measurement devices, such as measuring tapes, water scales and conventional theodolites $12,3,4,10 /$. With these devices measurement time can be shorter, accuracy better and the measuring task easier to accomplish.

The Electronics Laboratory of VTT has recently been developing some new measurement products and prototypes which could also have potential use in construction applications. The Acmeter is a versatile optical coordinate meter product 
(see Fig. 3a) designed especially for the accuracy management system Acman of Prometrics Ltd $/ 1,6 /$. The distance measurement is based on the time-of-flight principle and angle measurements are accomplished with two optical encoders. The measurement target aimed at with a HeNe-laser beam and the target can be a natural diffuse surface up to a measurement range of $30 \mathrm{~m}$. The accuracy is $1 \mathrm{~mm}$ in the measurement range of 3 $30 \mathrm{~m}$. The current product version has been used in the experimental part of this study.

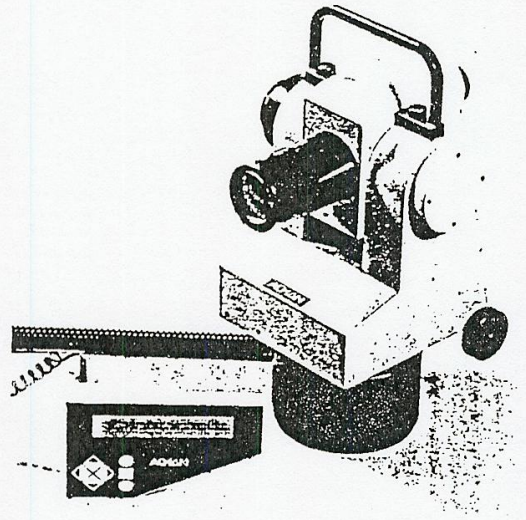

a) The Acmeter.

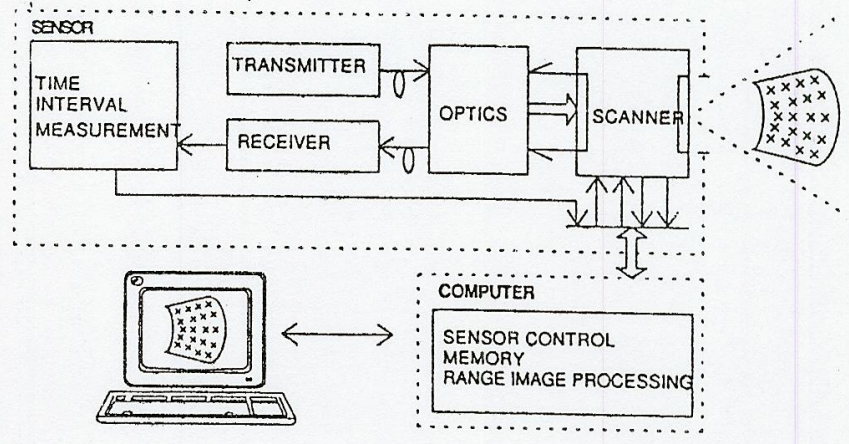

b) 3-D Vision System.

Figure 3. The equipment for 3-D measurement experiments.

The 3-D vision system prototype $/ 6,7 /$, developed by the Electronics Laboratory of VTT and the University of Oulu, is a laser scanning system in which distance measurement is similar to the above mentioned Acmeter. In the first prototype, a range image of the scene is created by scanning the laser in two directions with two mirrors. The measurement range of the system is $2.5-4.0 \mathrm{~m}$, the resolution is better than $3 \mathrm{~mm}$, the maximum image size is $512 \times 512$ and the measurement time is 10000 points/s. At the moment the new prototype is under final tests. Its structure is based on Acmeter and two servoed dc-motors used to direct the laser beam. It is planned to be the main device in our 3-D measurements of complicated objects. The principle of both prototypes of 3$\mathrm{D}$ vision system is presented in Fig. $3 \mathrm{~b}$.

\section{EXPERIMENTS}

In the study of 3-D measurements in construction, two special cases were selected. They were measurements in repair construction and framework erection. In this paper we present preliminary results of 3-D measurements in repair construction. First the previously presented concept was adapted to the task, see Fig. 4a. It is still on an abstract level, but it can be adapted to any real repair construction project. The major difference between the general concept and the one presented for repair construction is the absence of machines. This difference originates from our belief that most repair construction tasks in the near future will be manual. Also original data, object and the external measurement system have a more "down-to-earth" shape.

After the initial analysis several details of our laboratory building were modelled from the original drawings. They were created in our own graphics and measurement simulation programme. An example of a drawing of a simple wall produced by the demonstration system is in Fig. 4b. The cube in the middle of the picture marks the sensor. This model was also used to simulate the best way to carry out measurements. We used the Acmeter as a measurement device to provide 3-D data for the room. The 
Acmeter is composed of a 3-D coordinate meter and an integrated data system. The measurements were later compared with the model-based data of the room. These kind of measurements are needed in such repair construction tasks where drawings of the building are inaccurate or missing. For example, 3-D data is important during the planning of furnishing in order to avoid mismatching of new furniture.

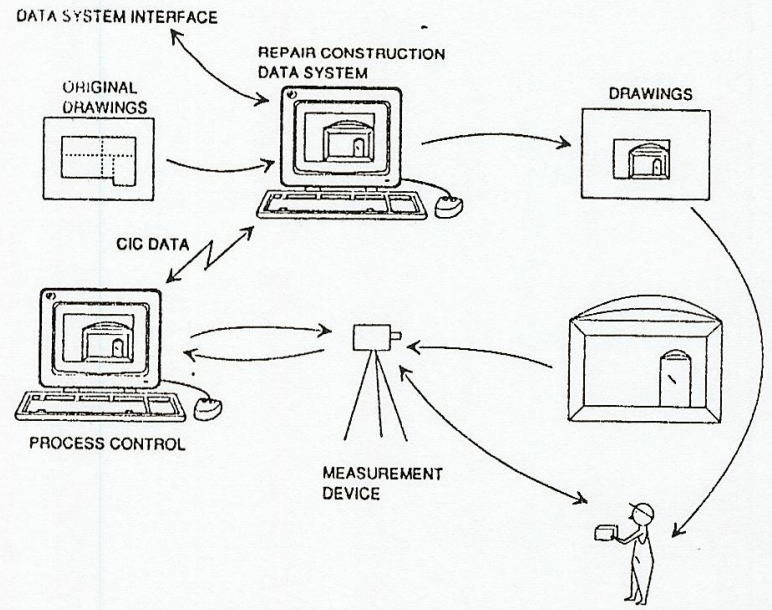

Figure 4. a) 3-D measurements.

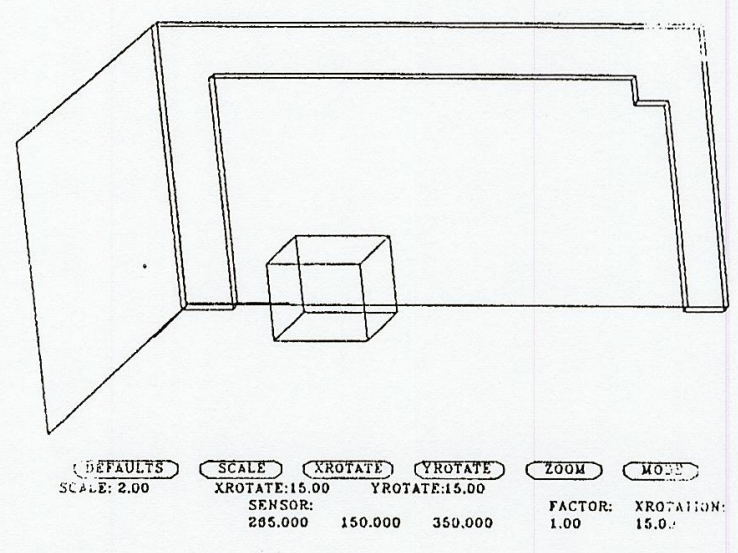

b) Modelled room wall.

This modest demonstration showed that 3-D measurements are applicable and the concept is adaptable to repair construction with off-the-shelf products. All the ingredients were integrated into one operating system, only the CIC data link between process control computer and repair construction data system was left out of the demonstration. The whole system could be built up quite easily. True, it would be still an experimental system and a great deal of work would be needed to find or make a practical modelling programme working in the PC environment. Also other details should be developed. For example, the Acmeter is currently manually operated. This means that instead of the automatical control of measurements, we have to transfer the measurement programme from the simulation to the measurement device manually.

\section{FURTHER RESEARCH AND DEVELOPMENT}

The use of the Acmeter in repair construction is limited to measurements of orderly surfaces such as planes and simple curved objects. Also the manual aiming of the device limits the measurements. In this respect the 3-D vision system (Fig. 3b) is more advanced. It can be used either as a programmable coordinate meter or a scanning device. For example, a previously planned and simulated measurement programme can be carried out easily. When the direction angles are fed to the device, it turns to the right direction and measures the $3-\mathrm{D}$ coordinate. Then the measurement programme can store, measure again or reject the result after which the operation can continue. There are many possibilities for the utilization of the properties of the 3-D vision system.

In Fig. 5 a task for a more demanding 3-D measurement is presented. This measurement of the curved surfaces of a clothes rack and four adjacent columns will be a next case study in the research. These kind of measurements are needed, e.g., in the repair construction of historically important buildings. The task could be carried out with a coordinate meter, but with the scanning laser the result will have more details. We intend to demonstrate the possibilities of the 3-D vision system in complicated repair construction tasks. This system is easily integrated with the experimental simulation and 
graphies programme, In principle, the 3-D measurements concept of repair construction, presented in Fig. 4, is fully utilized. One of the major problems to remain in this experimental part is the somewhat awkward interface between graphics and the results of measurements.
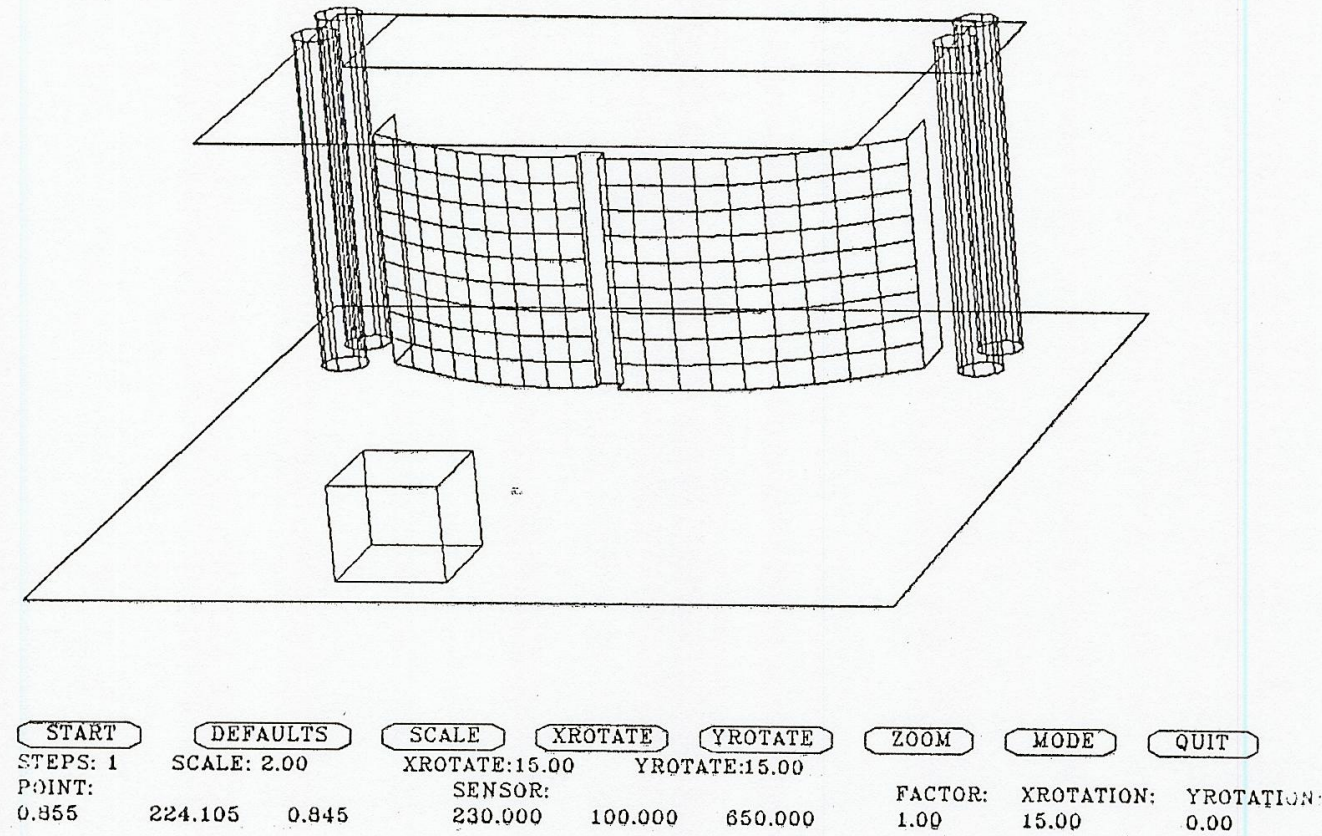

Figure 5. The uneven curved surface of a clothes rack.

\section{CONCLUSIONS}

At the moment the current measurement products can't provide ready solutions to computer integrated construction, complicated 3-D measurements and extensive robotization. The high prices and inconveniences of operation have restricted the use of advanced measurement products, but in the near future competition in the measurement product market will probably lower prices and make the new devices more suitable for a construction process.

The proposed general concept of using 3-D measurements is adaptable to most fields of construction. In particular, the experimental demonstration system for repair construction purposes shows its flexibility. It also demonstrates that a working solution for 3-D measurements depends on two major factors. The first (and the more important) factor is a mature and carefully designed measurement technology. The second factor is an operational concept to utilize advanced devices. There are a lot of possibilities to rationally adapt advanced technology to construction measurements. In this respect the importance of CIC and CAD is apparent.

\section{ACKNOWLEDGEMENTS}

The authors wish to thank Mr. Heikki Ailisto for his contribution to this work. 


\section{REFERENCES}

1. Acman - Manufacturing accuracy measurement and control system. Prometrics Ltd, Product presentation leaflet, 1988, Oulu, Finland.

2. Barry, B.A., Construction measurements, John Wiley \& Sons, 1988, 346 s.

3. Evans, J.M.,(ed.), Measurement technology for automation in construction and large scale assembly. Proc. of a Workshop, U.S. Department of Commerce, NBSIR 85-3210, Aug. 1985, 67 p.

4. Goodin, A.C., Boyer, L.T., Identification and measurement technologies applicable to construction. Proc. of the 4th Int. Symp. on Robotics and Artificial Intelligence in Building Construction, Haifa, Israel, 1987, pp. $863-877$.

5. Koskela, L., Lempinen, H., Salo, E., Wetlesen, T., The feasibility of construction robotics in Finland and Norway, The 6th Int. Symp. on Automation and Robotics in Construction, San Francisco, 6. - 8. June, 1989, pp. 80 - 86.

6. Kaisto, I., Kostamovaara, J., Moring, I., Myllylä, R., Laser rangefinding techniques in the sensing of 3-D objects, Sensing and Reconstruction of 3-D Objects and Scenes, SPIE Vol. 1260, Santa Clara, USA, 11. - 16. February, 1990, $12 \mathrm{p}$.

7. Moring, I. et al., Acquisition and processing of range data using laser-scanner based 3-D vision system. Proc. of the SPIE Conference on Optics, Illumination and Image Sensing for Machine Vision, Cambridge, MA, USA, November 1987, $10 \mathrm{p}$.

8. Ochi, T., Mio, K., A positioning system for mobile robots in construction applications ("Laser Positioner"). Proc. of the 5th Int. Symp. on Robotics in Construction, Tokyo, Japan, 1988, pp. $333-340$.

9. Pieskä, S., Lindholm, M., Koskela, L. Measurement requirements and advanced techniques for construction robotization. VTT research notes 1090, Espoo, January $1990,43 \mathrm{p}$.

10. Salo, E., Koskela, L., Construction Robotics. Present State and Views of Development. Espoo 1987, VTT, Tiedotteita 746, (in Finnish).

11. Shiokawa, T., Yamashita, Y., Tsuchiya, K., Navigation / positioning control of mobile robots for construction. Proc. of the 5th Int. Symp. on Robotics in Construction, Tokyo, Japan, 1988, pp. 589 - 598.

12. Ueno, T, et. al., Research \& development on robotic systems for assembly and finishing work. Proc. of the 5th Int. Symp. on Robotics in Construction, Tokyo, Japan, 1988, pp. $279-287$. 